\section{Amyand's hernia in a child with permanent neonatal diabetes due to pancreatic agenesis}

\author{
Alessandro Giannattasio,' \\ Riccardo Campus, ${ }^{2}$ Monica Muraca, \\ Giorgio Lucigrai, ${ }^{3}$ Alberto Michelazzi, ${ }^{2}$ \\ Etayeb Mahmud Eljerbi, ${ }^{3}$ Renata Lorini, \\ Giuseppe d'Annunzio' \\ 'Department of Pediatrics, University of \\ Genoa, Giannina Gaslini Institute, \\ Genova, Italy; '2Department of Pediatric \\ Surgery, Giannina Gaslini Institute, \\ Genova, Italy; ${ }^{3}$ Department of Pediatric \\ Radiology, Giannina Gaslini Institute, \\ Genova, Italy
}

\begin{abstract}
Acute or perforated appendicitis within inguinal hernia is rarely encountered and it is known as Amyand's hernia. We report on the first case occurring in a 4-year-old boy affected by permanent neonatal diabetes mellitus due to pancreatic agenesis, an extremely rare condition. The initial suspicion of inguinal hernia was confirmed by ultrasound examination of the right inguinal region which revealed omental layers inside a swollen inguinal canal; this finding and the clinical presentation allowed a prompt and appropriate surgical management. The careful evaluation of this patient and early recognition of this unique presentation of appendicitis allowed trans-hernial appendectomy and immediate herniorrhaphy. Ultrasonography played a pivotal role to reach the correct diagnosis and to start a prompt treatment.
\end{abstract}

\section{Introduction}

The finding of an uninflamed appendix in an inguinal hernial sac is estimated to be found in approximately $1 \%$ of adult inguinal hernia repairs; furthermore, it is three times more likely in children than in adults. ${ }^{1,2}$ The presence of a vermiform appendix in an inguinal hernial sac is termed Amyand's hernia $(\mathrm{AH})$, and has almost never been diagnosed prior to surgery. ${ }^{3,4}$ The first case of $\mathrm{AH}$ was described in 1735 by the surgeon Claudius Amyand and its incidence is estimated to range from 0.08 to $0.13 \%$ of all cases of appendicitis. ${ }^{5,6}$ To date, only few case reports have been published.

Neonatal diabetes mellitus (NDM) is a rare disorder affecting 1:400,000 newborns characterized by insulin-requiring hyperglycemia occurring within the first month of life; it can be classified into transient and permanent. ${ }^{7,8}$ Variable and complex mechanisms underlie this condition, and several genetic defects responsible for both forms have been identified, but only 19 cases due to pancreatic agenesis have been reported in the literature. ${ }^{9}$

\section{Case report}

A Caucasian boy born of non-consanguineous parents at 37 weeks of gestation, delivered by caesarean section, with severe intrauterine growth retardation (weight $1620 \mathrm{~g}$, length $45 \mathrm{~cm}$ ) developed severe hyperglycemia on the first day of life $(500 \mathrm{mg} / \mathrm{dL})$. NDM was diagnosed and treated with intravenous insulin infusion. Atrial septal defect was identified; no dysmorphic features were observed. Abdominal ultrasound revealed pancreas agenesis, then confirmed by magnetic resonance imaging. Karyotype analysis resulted normal (46 XY); other main laboratory data are summarized in Table 1. Enteral feeding was started with infant formula at $48 \mathrm{hrs}$ after birth and pancreatic enzyme therapy was administered due to pancreatic exocrine insufficiency. Screening for hypothyroidism revealed low fT4, low fT3, and increased TSH: L-thyroxine was started at a daily dose of $10 \mu \mathrm{g}$. Thyroid ultrasound revealed normal position, size and shape of the gland. Treatment with L-thyroxine was suspended at two years of age after retest of thyroid parameters which showed euthyroidism. Despite strict diabetes management by the parents and normal values of HblAc, glycemic values were extremely brittle with frequent spikes. Catch-up growth was observed at two years.

At 4.5 years of age the patient was admitted to our Emergency department because of fever, with right lower quadrant pain and induration. Physical examination revealed an acute irreducible swelling, hard and tender to palpation, located in the right inguinal region. Laboratory analysis showed leukocytosis in the full blood count. Urinalysis was normal. An obstructed inguinal hernia was initially diagnosed. Ultrasonography (US) examination revealed the right inguinal canal occupied by omental sheets, peritoneal fluid and a tubular elongated intestinal structure. This intestinal structure had a layered appearance, was about 2.5 mm thick and had a blind extremity. These findings were consistent with appendix. The right testicle was normal in the scrotum, surrounded by mild hydrocele (Figure 1).

Under general anesthesia, an incision was made in the skin crease of the right inguinal region, just above the superficial ring. The deep fascia was opened and the external oblique aponeurosis was identified and sepa-
Correspondence: Alessandro Giannattasio, Department of Pediatrics, University of Genoa, Giannina Gaslini Institute, largo Gerolamo Gaslini 5, 16147 Genova, Italy.

Phone: +39.010 .5636528 . Fax: +39.010 .93431133$

E-mail: alessandro@giannattasio.com

Key words: Amyand's hernia, neonatal diabetes, pancreas agenesis, ultrasonography

Contributions: all the authors contributed equally in the study.

Disclosures: all the authors certify that does not exist any financial or other relationship that could lead to a conflict of interest. The manuscript has been approved by all the authors.

Received for publication: 15 July 2009.

Revision received: 11 October 2009.

Accepted for publication: 12 October 2009.

This work is licensed under a Creative Commons Attribution 3.0 License (by-nc 3.0)

(C) Copyright A. Giannattasio et al., 2009

Pediatric Reports 2009; 1:e6

doi:10.4081/pr.2009.e6

rated to reveal the spermatic cord, where the hernial sac was identified and isolated. Upon opening the peritoneo-vaginal duct, the appendix and of the cecum were found to be lying within, with minimal adhesions to the sac. The cecum was freed from the flimsy adhesions to the sac and after a trans-hernial appendectomy, the caecum was reduced within the abdominal cavity. Then the surgeon performed an immediate herniorrhaphy. The patient had an uneventful post-operative period and was discharged after three days. The patient was treated with intravenous fluid and insulin and metabolic control was stable.

\section{Discussion}

Here we report on one of the few cases of $\mathrm{AH}$ occurring in a child, and absolutely the first case in a boy affected by permanent neonatal diabetes mellitus due to pancreatic agenesis., ${ }^{2,5}$

This condition appears to be caused by adhesions of the inflamed appendix occurring inside an enlarged hernial orifice rather than by external compression of the appendix base. Most of the cases occur on the right side, probably as a consequence of the normal anatomical position of the appendix and also because right-sided inguinal hernias are more common than left-sided hernias.

Because of the sequestered nature of the inflammatory process, $\mathrm{AH}$ presents with few classic systemic signs or symptoms suggestive of acute appendicitis; for this reason, in the 
Table 1. Clinical characteristics and main laboratory data of the patient.

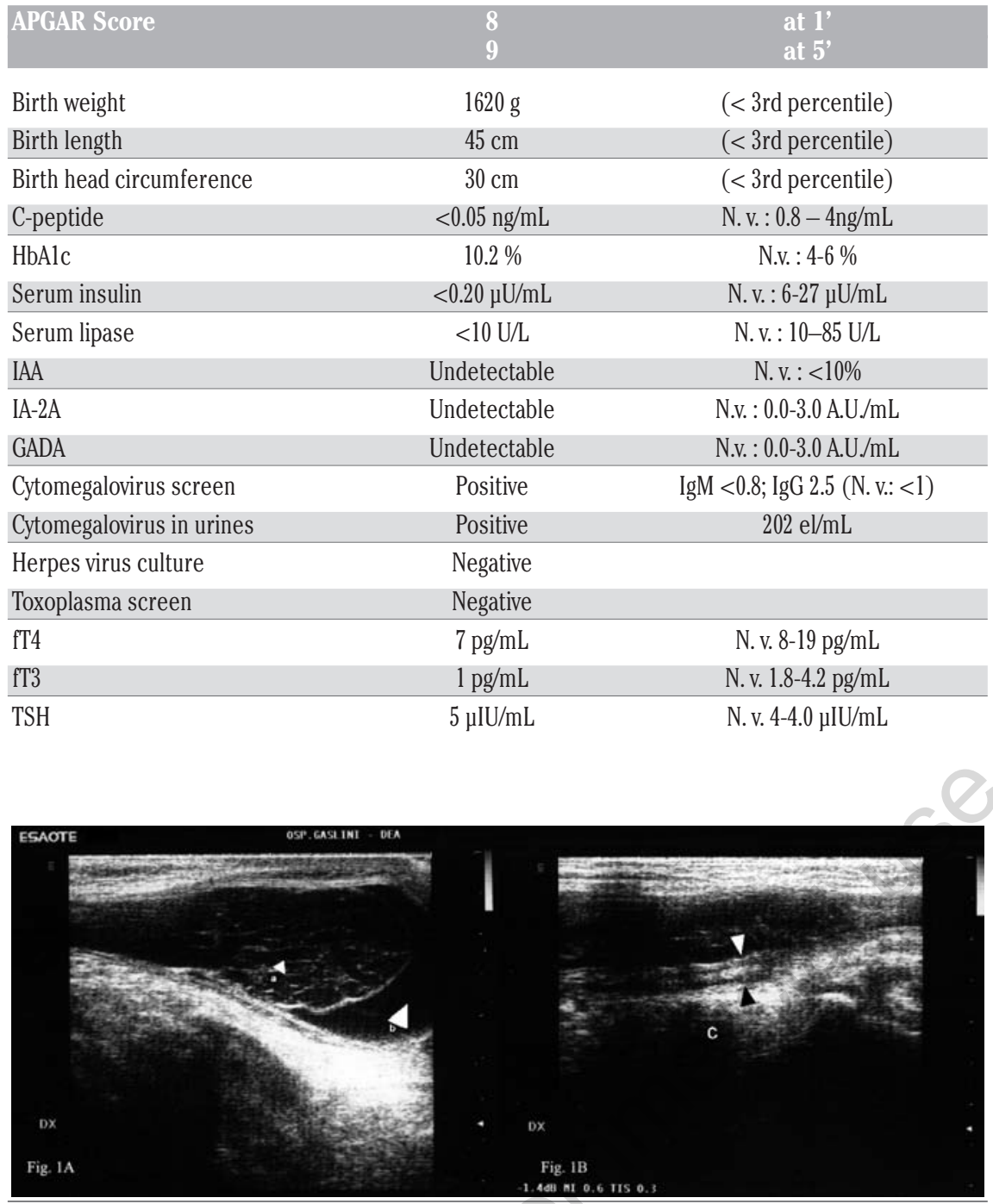

Figure 1. US longitudinal section of the right inguinal canal. A. The lumen of the canal is occupied by omental sheets (a) and peritoneal fluid (b). B. The appendix (arrowheads) is close to omental structure (c) with its typical layered intestinal appearance and its blind end.

past the diagnosis was nearly always made intra-operatively; pre-operative diagnosis is very difficult and only two authors reported a correct pre-operative diagnosis. ${ }^{10,11}$ Although pre-operative computed tomography (CT) of the abdomen may be helpful in reaching the correct diagnosis, the method of choice to study the inguinal canal is US examination. Availability, lack of invasiveness, no need of perfect patient immobilization and possibility to use it at the patient's bed, make US examination the method of choice for soft tissue imaging in pediatric patients. In particular, the new generation of high-definition small parts probes of ultrasonographic devices provides a better resolution than CT in the study of superficial small parts such as inguinal canals and appendix..$^{10,11}$
In our case, US examination allowed a diagnosis to be made, later confirmed intra-operatively after hernial sac opening. Early recognition of this unique presentation of appendicitis allows trans-hernial appendectomy and immediate herniorrhaphy. Delayed diagnosis requires drainage of the abscess with appendectomy and hernia repair. ${ }^{12}$ Prognosis depends on early and urgent surgical management while mortality rate is nearly zero.

In the literature, some studies revealed that insulin-like peptide 3 (Insl-3, a member of relaxin-insulin superfamily of hormones) has a putative role in a variety of physiological processes. In particular, it seems to play a role in the process of determination of the differential position of the gonads in male and female transgenic mice. In addition, Insl-3 expression causes inguinal hernia in female transgenic mice suggesting that a combined action of estrogens and Insl-3 disrupts proper development of muscular and connective tissue in abdominal structures: in our case, the abdominal wall muscle layer was thin, especially at the inguinal region. ${ }^{13}$

Congenital absence of the pancreas may be due to Ipf-1 (Insulin promoter factor-1) mutations: this condition could lead to an abnormal ectodermal development during pregnancy which involved other abdominal tissues like the omentum. ${ }^{14,15}$ Even if our patient resulted negative for Ipf-1 mutations, lack of fetal insulin production could lead to over expression of other insulin promoters like Insl-3 which influences collagen development of embryonal tissues; we suspect a similar condition could predispose to develop Amyand hernia. Congenital CMV, whose typical clinical symptoms may include intrauterine growth restriction (IUGR), microcephaly, hepatosplenomegaly, petechiae, jaundice, chorioretinitis, thrombocytopenia, anemia and/or other atypical findings, was excluded since the mother resulted IgG positive and IgM negative at CMV screen during pregnancy, while the newborn resulted both IgM and IgG positive showing a postnatal CMV infection. ${ }^{16}$

In conclusion, our case shows the importance of early diagnosis of $\mathrm{AH}$ which allowed prompt appropriate surgical treatment and avoided further complication. Therefore, we recommend particular attention when dealing with patients with abdominal malformations (like pancreatic agenesis) in case they present with abdominal hernia; US may play a pivotal role in reaching the correct diagnosis.

\section{References}

1. Gurer A, Ozdogan M, Ozlem N, Yildirim A, Kulacoglu H, Aydin R. Uncommon content in groin hernia sac. Hernia 2006; 10:152-5.

2. Yazici M, Etensel B, Gürsoy H, Ozkisacik S, Erkus M, Aydin ON. Infantile Amyand's hernia. Pediatr Int 2003; 45:595-6.

3. Hiatt JR, Hiatt N. Amyand's hernia. N Engl J Med 1988; 318:1402.

4. Logan MT, Nottingham JM. Amyand's hernia: a case report of an incarcerated and perforated appendix within an inguinal hernia and review of the literature. Am Surg 2001; 67:628-9.

5. D'Alia C, Lo Schiavo MG, Tonante A, et al. Amyand's hernia: case report and review of the literature. Hernia 2003; 7:89-91.

6. Sharma H, Gupta A, Shekhawat NS, Memon B, Memon MA. Amyand's hernia: a report of 18 consecutive patients over a 15 year period. Hernia 2007; 11:31-5.

7. Polak M, Cavé H. Neonatal diabetes mellitus: a disease linked to multiple mecha- 
nisms. Orphanet J Rare Dis 2007; 2:12.

8. Shield JP. Understanding neonatal diabetes mellitus. J Pediatr 2002; 141: 462-3.

9. Chen R, Hussain K, Al-Ali M et al. Neonatal and late-onset diabetes mellitus caused by failure of pancreatic development: report of 4 more cases and a review of the literature. Pediatrics 2008; 121:e1541-7.

10. Ash L, Hatem S, Ramirez GA, Veniero J. Amyand's hernia: a case report of prospective ct diagnosis in the emergency department. Emerg Radiol 2005; 11:231-2.

11. Coulier B, Pacary J, Broze B. Sonographic diagnosis of appendicitis within a right inguinal hernia (Amyand's hernia). J Clin Ultrasound 2006, 34:454-57.

12. Weber RV, Hunt ZC, Kral JG. Amyand's hernia: Etiologic and therapeutic implications of two complications. Surg Rounds 1999, 22:552-6.

13. Koskimies P, Suvanto M, et al. Female mice carrying a ubiquitin promoter-Insl3 transgene have descended ovaries and inguinal hernias but normal fertility. Mol Cell Endocrinol 2003; 206: 159-66.

14. Shabanpoor F, Bathgate RA, Hossain MA, et al. Design, synthesis and pharmacological evaluation of cyclic mimetics of the insulin-like peptide 3 (INSL3) B-chain. J Pept Sci 2007; 13:113-20.

15. Stoffers DA, Zinkin NT, Stanojevic V, et al. Pancreatic agenesis attributable to a single nucleotide deletion in the human IPF1 gene coding sequence. Nat Genet 1997;15:106-10.

16. Ornoy A, Diav-Citrin 0. Fetal effects of primary and secondary cytomegalovirus infection in pregnancy. Reprod Toxicol 2006;21:399-409. 